\title{
SCIDOC
}

International Journal of HIV/AIDS and Research (IJHR)

ISSN: 2379-1586

\section{Effect of Body Weight on Cytokine Response to Anti-tubercular Therapy in Patients with HIV Infection and Tuberculosis}

Research Article

Ivaturi Venkata Nagesh ${ }^{1 *}$, Sashindran $\mathrm{VK}^{2}$, Anurag Sonkhle ${ }^{3}$

${ }^{1}$ Professor (Internal Medicine), MH Ambala, Haryana, India.

${ }^{2}$ Professor and Consultant (Internal Medicine \& Infectious Diseases), Principal Medical Officer, Central Air Command, Prayagraj, Uttar Pradesh, India.

${ }^{3}$ Resident(Internal Medicine), AFMC, Pune, India.

\section{Abstract}

Introduction: Nutrition plays an important role in stimulating the immune response to varied pathogens especially in patients with tuberculosis. Cytokine response to antitubercular therapy is highly variable in patients with HIV infection and Nutrition plays an important role in determining this response

Objectives: To study the effect of body mass index in the levels of cytokines IL-10, IP-10, IL-4 and Interferon- Gamma before and after treatment with anti tuber-cular therapy in patients with HIV infection

Material and methods: It is a prospective cross sectional study, with a population of 50 individuals with HIV infection with tuberculosis were studied.

Results: The mean levels of cytokines IFN-Gamma, IP-10, IL-10 and IL-4 in low BMI group before initiation of ATT are $72.62 \mathrm{U} / 1,8.90 \mathrm{pg} / \mathrm{ml}, 26 \mathrm{pg} / \mathrm{ml}$ and $1.24 \mathrm{pg} / \mathrm{ml}$. The levels after completion of 02 months of ATT are 17.10, $4.56,9.14$ and 0.57 respectively.

The mean levels of cytokines IFN-Gamma, IP-10, IL-10 and IL-4 in normal BMI group before initiation of ATT are 22.32, 4.17, 11.25 and 0.78 . The levels after completion of 02 months of ATT are 17.10, 4.56, 9.14 and 0.57 respectively.

Conclusions: BMI as surrogate marker for nutrition pals an important role in cytokine response. The change in levels of Interferon - gamma in low BMI group were statistically significant.

Keywords: Cytokines; Tuberculosis; HIV Infection, Body Mass Index.

\section{Introduction}

Tuberculosis is one of the major public health issues across the world and remains the leading cause of death worldwide. Tuberculosis is the commonest opportunistic infection in people with $\mathrm{HIV} / \mathrm{AIDS}$ and the combination is lethal. According to WHO Report 2018, about 2,51,000 people died of HIV-associated TB (HIV-TB). In 2018, there were an estimated 8,62,000 new cases of TB amongst people who were HIV-positive [1]. India accounts for about $10 \%$ of the global burden of HIV-TB.

Nutrition has a vital role in the immune functions of an individual. HIV-TB is known to cause wasting in the patients. WHO recognises Tuberculosis and HIV to be associated with caloric, protein malnutrition, micronutrients deficits and recommends the patients with mild or moderate malnutrition to eat a healthy diet that meets th Recommended Dietary Allowance (RDA) [2].

Malnutrition, as indicated by body mass index (BMI), is the leading cause of sec-ondary immune deficiency and is known to have a direct effect on T- Lymphocyte response. It is also associated with reduced cytokine response. The level of circulat-ing pro-inflammatory cytokines are reduced whereas the level of regulatory cytokines are increased in an individual with the low nutritional status [3]. Many animal studies have shown that malnutrition has a detrimental effect on host immunity against infection. Lack of proteins and other nutrients in the diet causes thymic atrophy and impairs generation, maturation and function of $\mathrm{T}$ - Lymphocytes. Protein deficiency also impairs sequestration of reactive T lymphocytes thereby causing loss of tuberculosis resistance and

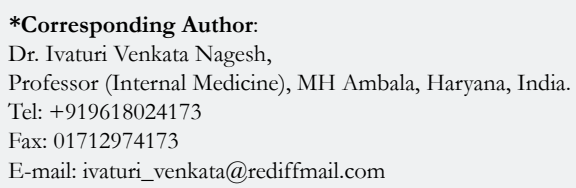

Citation: Ivaturi Venkata Nagesh, Sashindran VK, Anurag Sonkhle. Effect of Body Weight on Cytokine Response to Anti-tubercular Therapy in Patients with HIV Infection and Tuberculosis. Int J AIDS Res. 2020;7(1):196-199. doi: http://dx.doi.org/10.19070/2379-1586-2000037

Copyright: Ivaturi Venkata Nagesh ${ }^{\circ} 2020$. This is an open-access article distributed under the terms of the Creative Commons Attribution License, which permits unrestricted use, distribution and reproduction in any medium, provided the original author and source are credited. 
also makes the monocytes macrophages to produce TGF $-\beta$, known as a mediator of immunosuppression in tuberculosis [4]. The role of nutrition in cytokine response of an individual suffering from HIV-TB is compounded by the direct effect of HIV on the cytokine levels. HIV infection leads to a chronic inflammatory state and is associated with increase in levels of proinflammatory cytokine like IFN $\gamma$ and reduced levels of IL-6. Whereas on the other hand malnutrition blunts the immune inflammatory response as characterized by reduced levels of pro-inflammatory cytokines like IFN $\gamma$ and raised levels of IL-6 and IL-4 [8]. Th1 cytokines required for Th1 differentiation (IL-7, IL-12, IL-18 and IL-21) and function (IL-2 and IFN- $\gamma$ ) are reduced in peripheral blood mononuclear cells of children with malnutrition and severe infection. In the same children, an overexpression of Th2 cytokines (IL-4 and IL-10) and increased apoptosis of CD3+ T cells is noted [9]. Hence we planned to study the effect of body mass index on two pro-inflammatory cytokines i.e interferongamma(IFN - $\gamma$ ), and interferon gamma inducing protein -10 (IP10), and two anti-inflammatory cytokines: interleukin-4(IL-4) and interleukin-10(IL-10).

Aim: To assess the role of malnutrition on the cytokine response to anti -tubercular therapy (ATT) in HIV-TB.

\section{Objectives:}

1) To assess the effect of BMI on cytokine levels prior to starting ATT.

2) To assess the effect of BMI on cytokine levels after intensive phase of ATT.

3) To determine if nutritional status has a role in cytokine response to ATT (i.e. change in levels).

\section{Material and Methods}

In this prospective pilot study all consenting patients of HIV-TB with either fresh pulmonary/extra-pulmonary TB being started on ATT were enrolled. Over the study duration 50 patients were enrolled and followed till the completion of intensive phase (IP) of ATT (i.e. for 2 months). The exclusion criteria were as follows:

\section{- Patients on long- term steroids}

- History of diabetes mellitus, rheumatological disorders

- Past history of tuberculosis

- Patients with chronic kidney disease

- Patients with acute viral infection (other than HIV)

- Patients with haematological malignancy

- Pregnancy

- Other opportunistic infections in patients with HIV infection

\section{Methodology}

Serum cytokine levels of IL-4, IL-10, IP-10 and IFN- $\gamma$ were estimated by ELISA, at the start of therapy (or within 02 weeks of initiation of ATT) and after 02 months of therapy.

\section{Serum Sample Processing}

Serum concentrations of IFN- $\gamma$, IL-4, IP-10 and IL-10 were determined by commercial ELISA kits (human IFN- $\gamma$, IL-4, and IL-10, IP-10) high sensitivity ELISA kits, Gen-Probe Diclone,
France. All tests were performed in duplicate.

\section{Statistical Analysis}

The statistical analysis of results was done by IBM SPSS statistics 22 software, and scatter plot along with linear regression analysis was done using statsmodels package in Python 3.7.

\section{Results}

The study was conducted on 50 patients with HIV-TB commencing ATT. Out of 50 patients, 43 were male and 7 were female. The average age was 39.42 years \pm 12.72 (SD). The mean duration of HIV illness was 2.4 years. The mean weight of the subjects was $57.10 \mathrm{~kg} \pm 11.07$ (SD). The mean values of haemoglobin (Hb), erythrocyte sedimentation rate (ESR) and CD4 cell count were $10.53 \mathrm{gm} / \mathrm{dl} \pm 1.65(\mathrm{SD}), 47.28 \mathrm{~mm}$ fall in $1 \mathrm{st} \mathrm{hr} \pm 18.93(\mathrm{SD})$ and 341.76 cells $/ \mathrm{cmm}$ respectively. Body Mass Index (BMI), the surrogate marker for malnutrition, was calculated and the patients were divided into three groups, individuals who were malnourished as reflected by low BMI $\left(<18.5 \mathrm{~kg} / \mathrm{m}^{2}\right)$, individuals who had normal nourishment status as reflected by normal BMI (18.5$24.9 \mathrm{~kg} / \mathrm{m}^{2}$ ) and individuals who were found to be overweight or obese during the study as indicated by BMI $\left(>24.9 \mathrm{~kg} / \mathrm{m}^{2}\right)$. Out of 50 patients 15 were underweight (BMI <18.5), 30 were with normal BMI (BMI 18.5 - 24.9) and 5 patients were overweight (BMI $>25.0 \mathrm{~kg} / \mathrm{m}^{2}$ ). The mean $\mathrm{Hb}$ level among this group was $9.14 \mathrm{~g} / \mathrm{dL}$, indicative of anaemia in these patients. The mean $\mathrm{Hb}$ of subjects with BMI $>18.5 \mathrm{~kg} / \mathrm{m}^{2}$ was $11.09 \mathrm{gm} / \mathrm{dl}$ which was higher as compared to the low BMI group. The mean cytokine values both pre-ATT and post-IP with respect to BMI are shown in Table 1 below.

The log cytokine levels were analysed before and after the intensive phase of ATT and paired 'T' test was performed. The values are shown in Table 2 below:

In order to analyse the effect of BMI on the cytokine levels, both at baseline and after the IP, BMI was considered as a continuous variable and multivariate linear regression analysis was done with BMI and treatment status (i.e. pre-ATT and post-IP). Strata scatter plot with overlying regression for all the cytokines is given in Figures 1- 4.

Linear regression analysis of $\log$ cytokines with time and BMI revealed log IP10 is inversely related to BMI and log IFN gamma significantly decreased after treatment as given in Table 3 .

\section{Discussion}

Nutrition is an important component of treatment protocol in various acute and chronic medical ailments [10]. Various studies have revealed that malnutrition causes atrophic changes in the thymus gland resulting in sub-optimal T-cell proliferation and leading to secondary immunodeficiency $[5,9]$. HIV infection further impairs cell-mediated immunity and down-regulates immune response thereby predisposing these patients to many opportunistic infections [6].

As BMI has been taken as surrogate marker of nutrition in our study, the role of the nutrition cannot be overlooked in popula- 
Table 1.

\begin{tabular}{|c|c|c|c|c|c|c|c|c|}
\hline \multirow{2}{*}{} & \multicolumn{2}{|c|}{$\begin{array}{c}\text { IFN }-\boldsymbol{\gamma} \\
\text { Mean(SD) }\end{array}$} & \multicolumn{2}{c|}{$\begin{array}{c}\text { IP-10 } \\
\text { Mean(SD) }\end{array}$} & \multicolumn{2}{c|}{$\begin{array}{c}\text { IL-10 } \\
\text { Mean(SD) }\end{array}$} & \multicolumn{2}{c|}{$\begin{array}{c}\text { IL-4 } \\
\text { Mean(SD) }\end{array}$} \\
\cline { 2 - 9 } & Pre & Post & Pre & Post & Pre & post & Pre & Post \\
\hline$<18.5$ & 72.62 & 17.1 & 8.9 & 4.56 & 26 & 9.14 & 1.24 & 0.57 \\
\hline $\mathrm{kg} / \mathrm{m} 2$ & -127.08 & -28.29 & -13.25 & -13.09 & -36.53 & -6.66 & -0.67 & -0.36 \\
\hline$>18.5$ & 23.32 & 9.54 & 4.17 & 2.58 & 11.25 & 9.18 & 0.78 & 0.61 \\
\hline $\mathrm{kg} / \mathrm{m} 2$ & -25.09 & -12.42 & -6.81 & -4.52 & -8.14 & -0.78 & -0.46 & -0.36 \\
\hline
\end{tabular}

Table 2.

\begin{tabular}{|c|c|c|c|c|c|c|c|c|c|}
\hline Log Cytokine & \multicolumn{4}{|c|}{ Pre-ATT } & \multicolumn{4}{c|}{ Post-IP } & \\
\hline & Mean & Median & SD & Range & Mean & Median & SD & Range & $\begin{array}{c}\text { T Test } \\
\text { p value }\end{array}$ \\
\hline IFN - $\gamma$ & 2.81 & 2.728 & 0.91 & $2.33-3.35$ & 1.761 & 1.988 & 1.21 & $1.58-2.38$ & 0.001 \\
\hline IP-10 & 1.48 & 1.482 & 1.13 & $1.05-2.00$ & 1.16 & 1.203 & 1.298 & $0.55-1.81$ & 0.343 \\
\hline IL-10 & 2.288 & 2.343 & 0.87 & $2.02-2.77$ & 2.129 & 2.385 & 1.03 & $1.89-2.72$ & 0.546 \\
\hline IL-4 & -0.316 & -0.209 & 0.78 & -1.09 & -0.701 & -0.513 & 0.86 & -0.857 & 0.095 \\
\hline
\end{tabular}

Table 3.

\begin{tabular}{|c|c|c|c|c|}
\hline Variance & Index & coef & 1cl_95 & ucl_95 \\
\hline \multirow{2}{*}{$\log$ _IFN $-\gamma$} & time [T.1] & -1.0499 & -1.6418 & -0.4579 \\
\cline { 2 - 5 } & bmi & 0.0013 & -0.0802 & 0.0828 \\
\hline \multirow{2}{*}{ log_IP-10 } & time [T.1] & -0.2099 & -0.8672 & 0.4472 \\
\cline { 2 - 5 } & bmi & -0.0971 & -0.1877 & -0.0066 \\
\hline \multirow{2}{*}{ log_IL-4 } & time [T.1] & -0.3519 & -0.8135 & 0.1096 \\
\cline { 2 - 5 } & bmi & -0.0295 & -0.0931 & 0.0340 \\
\hline \multirow{2}{*}{$\log$ _IL-10 } & time [T.1] & -0.1218 & -0.6543 & 0.4107 \\
\cline { 2 - 5 } & bmi & -0.0329 & -0.1063 & 0.0404 \\
\hline
\end{tabular}

Figure 1-4.
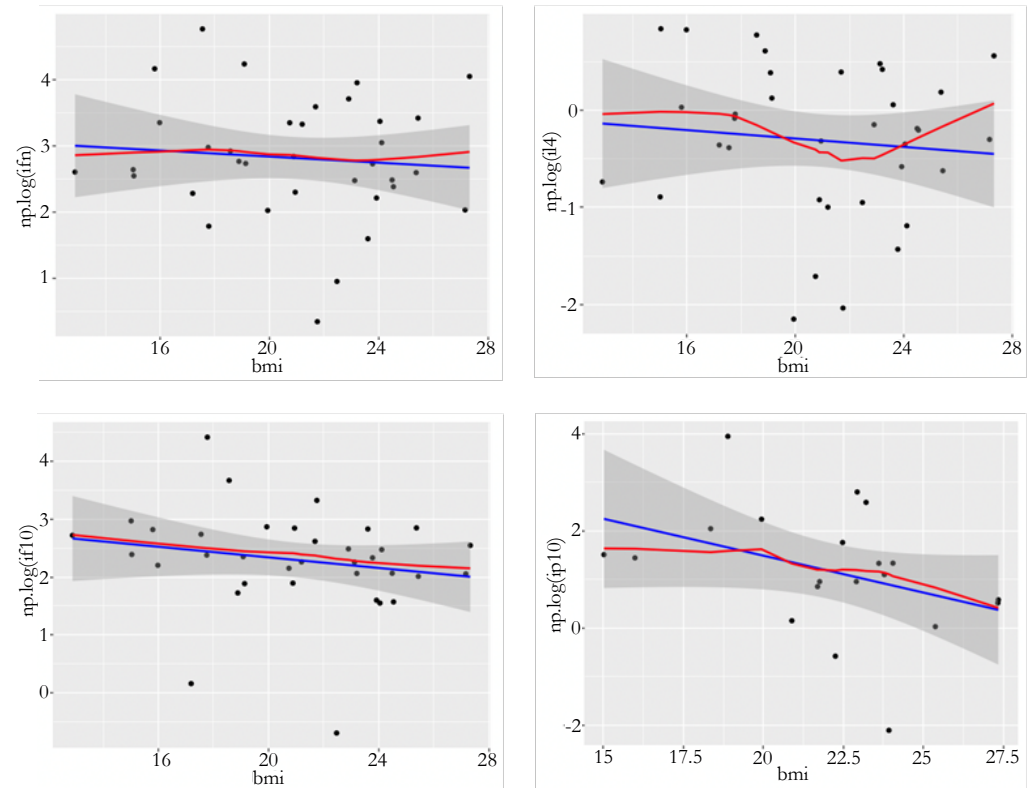

tion with poor nutritional status and low CD4 count. Lonnorth et. al [13] demonstrated that BMI is a reliable marker for nutritional status and there is $14 \%$ decrease in incidence of Tuberculosis with one unit rise in BMI. Leung that low BMI increases TB burden [14] and low BMI is associated with decrease in proinflammtory cytokines [11]. It has been demonstrated that immune response in individuals with low BMI is down regulated. The same is mani- fested in form of elevated levels of regulatory cytokines and reduced levels of pro-inflammatory cytokines, thereby increasing the susceptibility to acquisition of new infection and promoting disseminated disease in affected individuals [13].

In our study there were more no.of low BMI patients than normal. It is comparable to the study done by villamor [7] made who 
found similar results of more no. of malnourished indi-viduals than normal BMI patients in comparison to other countries. In our study we found the levels of pro inflammatory cytokines (IFN-gamma and IP-10) were high in low BMI group than that of normal BMI groups prior to initiation of ATT which is variance with other studies by Anuradha et al., [10, 11]. The reason may be due to other factors like higher disease burden of HIV and concomitant low CD4 counts which dominated over nutrition per se. The levels of anti inflammatory cytokine IL-10 levels were high in low BMI group than normal BMI group which is consistent with various studies [6, 7]. The decreased IL-10 levels may lead to down regulation of initial inflammatory response thereby promoting higher disease burden and disseminated disease as compared to individuals with normal BMI [7]. The low level of anti inflammatory cytokine levels may also due to HIV infection per se which is by nature chronic inflammatory condition leading to elevated levels of pro inflammatory cytokines and hence the higher levels of pro inflammatory cytokine levels despite low BMI are attributed to the same [9]. Our study revealed that the level of anti inflammatory cytokines IL-10 and IL-4 both at the baseline and after two months of ATT therapy are slightly higher than that of individuals with normal BMI. The levels of Interferon $\gamma$ levels were reduced from initial stage to 02 months after ATT therapy which was statistically significant, as was observed in linear regression analysis, similar to study done by Azzuri [15] but is in variance to study by Riou et. al [16]. However Riou et al measured Interferon $\gamma$ levels after 6 months of ATT, that too in HIV negative individuals. Since, most of our patients are on HAART the results were different because of decrease of viral load. A study by Mihret [17] found no significant change in levels of Interferon gamma or any other cytokine levels after treatment in HIV positive individuals. In our study the IP-10 levels were not reduced significantly after 02 months of anti tubercular therapy similar to the results by Mave [18] who noticed that the chemokine levels IP-10 were decreased only after 48 weeks of Anti retroviral therapy.

Logical aggression analysis revealed Log IP-10 values are inversely related to BMI in response to anti tubercular therapy in contrast to study by Azuri et.al [15] may be due to poor nutrition related to low level of pro inflammatory cytokine. In our study none of the anti inflammatory cytokines (IL-4, IL-10) showed a significant change before and after treatment simiilar to result by Mihret. However Siwaya et.al [19] found the levels of anti inflammatory cytokines low before initiation of treatment. This finding may suggest that in dually infected subjects, the HIV-related changes dominate the overall immunological pic-ture and leads to dysregulation of cytokine and chemokine production.

\section{Conclusion}

In our study anti inflammatory cytokines IL- 10 and IL-4 at the baseline as well as after two months of ATT therapy are slightly higher than that of individuals with nor-mal BMI. Interferon $\gamma$ levels were reduced from initial stage to 02 months of ATT therapy. This study demonstrated BMI plays an important role in change in the le-vels of cytokines which need larger studies to corroborate the findings.

\section{Acknowledgement}

We are grateful to Col Suman Kumar Pramanik, Professor Medicine and Haematology, Army Hospital (RR) New Delhi110010 for the statistical analysis of data.

\section{References}

[1]. Tuberculosis (TB) [Internet]. Who.int. 2020 [cited 26 March 2020]. Available: https://www.who.int/news-room/fact-sheets/detail/tuberculosis.

[2]. WHO. Guideline: nutritional care and support for patients with tuberculosis. 2013.

[3]. Rajamanickam A, Saravanan Munisankar, Yukthi Bhootra, Nathalla Pavan Kumar, Chandrakumar Dolla, Paul Kumaran, et al. Coexistent Malnutrition is associated with Pertubations in Systemic and Antigen - Specific Cytokine Responses in Latent Tuberculosis Infection. Clinical and Vaccine Immunology 2016; 23(4): 339-345. PMID: 26865593.

[4]. Dai G, McMurray DN. Altered cytokine production and impaired antimycobacterial immunity in protein - malnourished guinea pigs. Infect Immun.1998; 66: 3562 - 3568. PMID: 9673234

[5]. Savino W. The thymus gland is a target in malnutrition. Eur J Clin Nutr. 2002; 56(3): S46-49. PMID: 12142962.

[6]. McMurray DN. Cellular immune changes in undernourished children. Prog Clin Biol Res. 1981; 67: 305-318.

[7]. Villamor E, Saathoff E, Mugusi F, Bosch RJ, Urassa W, Fawzi WW. Wasting and body composition of adults with pulmonary tuberculosis in relation to HIV-1 co-infection, socio-economic status, and severity of tuberculosis. Eur J Clin Nutr. 2006; 60(2): 163-171. PMID: 16234841.

[8]. De Milito A. B lymphocyte dysfunctions in HIV infection. Current HIV Research. 2004; 2(1):11-21. PMID: 15053337.

[9]. Sashindran VK, Thakur R. Malnutrition in HIV/AIDS: Aetiopathogenesis. InNutrition and HIV/AIDS-Implication for Treatment, Prevention and Cure 2020 Feb 20. Intech Open.

[10]. Anuradha R, Munisankar S, Bhootra Y, Kumar NP, Dolla C, Kumaran P, et al. Coexistent malnutrition is associated with perturbations in systemic and antigen-specific cytokine responses in latent tuberculosis infection. Clin Vaccine Immunol. 2016; 23:339-345. PMID: 26865593.

[11]. Anuradha R, Munisankar S, Bhootra Y, Dolla C, Kumaran P, Babu S. High body mass index is associated with heightened systemic and mycobacterial antigen specific pro-inflammatory cytokines in latent tuberculosis. Tuberculosis. 2016; 101: 56-61. PMID: 27865399.

[12]. Chandrasekaran P, Saravanan N, Bethunaickan R, Tripathy S. Malnutrition: Modulator of Immune Responses in Tuberculosis. Front. Immunol. 2017; 8: 1316. PMID: 29093710.

[13]. Lonnroth K, Williams BG, Cegielski P, Dye C. A consistent log-linear relationship between tuberculosis incidence and body mass index. Int J Epidemiol. 2010; 39(1): 149-55. PMID: 19820104.

[14]. Leung CC, Lam TH, Chan WM, Yew WW, Ho KS, Leung G, et al. Lower risk of tuberculosis in obesity. Arch Intern Med 2007; 167(2): 1297-1304. PMID: 17592104

[15]. Azzurri A, Sow OY, Amedei A, Bah B, Diallo S, Peri G, et al. IFN-gammainducible protein 10 and pentraxin 3 plasma levels are tools for monitoring inflammation and disease activity in Mycobacterium tuberculosis infection. Microbes Infect. 2005; 7(1): 1-8. PMID: 15716076.

[16]. Riou C, Perez Peixoto B, Roberts L, Ronacher K, Walzl G, Manca C, et al. Effect of standard tuberculosis treatment on plasma cytokine levels in patients with active pulmonary tuberculosis. PLoS One. 2012; 7(5): e36886. PMID: 22606304.

[17]. Mihret A, Abebe M, Bekele Y, Aseffa A, Walzl G, Howe R. Impact of HIV co-infection on plasma level of cytokines and chemokines of pulmonary tuberculosis patients. BMC Infect Dis. 2014; 14: 125. PMID: 24592945.

[18]. Mave V, Erlandson KM, Gupte N, Ashwin Balagopal, David M Asmuth, Thomas B Campbell, et al. Inflammation and Change in Body Weight With Antiretroviral Therapy Initiation in a Multinational Cohort of HIV-Infected Adults. J Infect Dis. 2016; 214(1): 65-72. PMID: 26962236.

[19]. Siawaya J, Beyers N, van Helden P, G Walzl. Differential cytokine secretion and early treatment response in patients with pulmonary tuberculosis. J Infect Dis. 2009, 156: 69-77. PMID: 19196252. 